\title{
MRI T2-Weighted Cochlear Intensity as a Predictor of Hearing Loss with Vestibular Schwannoma Patients
}

\author{
${ }^{1}$ Department of Otolaryngology, Case Western Reserve University \\ School of Medicine, Cleveland, Ohio, United States \\ 2Department of Otolaryngology-Head and Neck Surgery, University \\ Hospitals Cleveland Medical Center, Cleveland, Ohio, United States \\ ${ }^{3}$ Department of Neurosurgery, University Hospitals Cleveland \\ Medical Center, Cleveland, Ohio, United States
}

Peter Q. Luong1 Claudia I. Cabrera ${ }^{2}$ Nirav Patil ${ }^{2}$ Hammad Khan ${ }^{1}$ Carlito Lagman ${ }^{3}$

Thomas A. Ostergard ${ }^{3}$ Gary Huang ${ }^{2}$ Nicholas C. Bambikidas ${ }^{3}$ Sarah E. Mowry ${ }^{2}$

Address for correspondence Sarah E. Mowry, MD, 11100 Euclid Avenue, LKS 4052, Cleveland, OH 44106, United States (e-mail: Sarah.Mowry@UHhospitals.org).

Ann Otol Neurotol ISO 2021;4:19-25.

\begin{abstract}
Keywords

- vestibular schwannoma

- hearing

$-\mathrm{MRI}$

- acoustic neuroma

Objective It is difficult to predict how hearing loss will progress with vestibular schwannomas (VSs) and to determine the optimal time for hearing preservation interventions. This study investigated the relationship between cochlear intensity on T2-weighted magnetic resonance imaging and hearing loss in VS patients over time.

Design Retrospective cohort study.

Setting Single major academic center.

Participants Patients with a diagnosis of VS from 2007 to 2018.

Main Outcome Measures Pure tone average (PTA) and cochlear-to-pontine relative signal intensity (RSI) measured at two time points.

Results Fifty patients were included in the final analysis. For both affected and unaffected ears, the trend in PTA increased from baseline to follow-up. For affected ears, the trend in RSI increased from baseline to follow-up, while for the unaffected ears RSI decreased. There was a significant positive correlation between the initial RSI value in the ipsilateral ear when compared with the change in PTA from baseline to follow-up $(r=0.28, p=0.048)$.

Conclusion There is a trend between initial RSI and how hearing changes in the affected ears of patients with VS. Additional studies are needed to explore how this relationship may be better used to predict hearing loss.
\end{abstract}

\section{Introduction}

Vestibular schwannomas (VSs) are benign tumors that typically develop from the eighth cranial nerve. Patients typically present with symptoms including hearing loss, tinnitus, dizziness, and vertigo. ${ }^{1}$ Magnetic resonance imaging (MRI) is the preferred imaging modality for diagnosis and screening after presentation of hearing loss. Changing patterns for imaging referrals and advances in MRI technology have resulted in an

Published online

September 14, 2021
DOI https://doi.org/

$10.1055 / \mathrm{s}-0041-1735394$

ISSN 2581-9607 increasing proportion of these tumors being found incidentally in patients with minimal or no symptoms. ${ }^{2}$

The management of larger tumors typically involves surgical excision or stereotactic radiosurgery, while smaller tumors, especially in asymptomatic patients, may be managed conservatively with observation and serial MRIs. ${ }^{3}$ The goal of observation is to avoid treatment until there is tumor growth and/or symptom progression.
(C) 2021. Indian Society of Otology.

This is an open access article published by Thieme under the terms of the Creative Commons Attribution-NonDerivative-NonCommercial-License, permitting copying and reproduction so long as the original work is given appropriate credit. Contents may not be used for commercial purposes, or adapted, remixed, transformed or built upon. (https://creativecommons.org/licenses/by-nc-nd/4.0/).

Thieme Medical and Scientific Publishers Pvt. Ltd. A-12, 2nd Floor, Sector 2, Noida-201301 UP, India 
A challenge for clinicians implementing this type of conservative management is the difficulty predicting when hearing loss will progress and when is the optimal time for intervention. ${ }^{4}$ While numerous associations have been made regarding tumor characteristics and imaging, there is to date no single best predictor for determining a patient's audiologic outcomes.

This study intended to find an association between patients' cochlear signal on T2 MRI imaging and their hearing, with the hope that an association will be able to help clinicians decide if and when definitive microsurgical hearing sparing intervention is warranted to attempt to save the hearing.

\section{Materials and Methods}

A retrospective chart review was conducted on patients diagnosed with a VS between 2007 and 2018 at a single major academic medical center. This study was approved by the hospital institutional review board. Search for records was conducted using International Classification of Diseases (ICD)-9 code 225.1 and ICD-10 code D33.3.

Patients were excluded on the based on the following: (1) being younger than 18 years of age, (2) insufficient data as to preclude complete radiographic and audiometric assessments, (3) neurofibromatosis type II, (4) having had surgical or radiosurgical intervention for their tumor, and (5) other prior history of other major otological conditions or surgery which could impact on their hearing outcomes (chronic otitis media, otosclerosis, etc.).

Variables collected included age, gender, baseline (earliest available ensured to precede any treatment), and most recent follow-up MRI and audiometry. For both baseline and follow-up data, the MRI and audiogram were ensured to be within 3 months of each other.

MRI was performed using an institutional specific MRI internal auditory canal (IAC) protocol. MR image analysis was performed using a SECTRA picture archiving and communication system. Two sets of images were viewed and analyzed: baseline images and the most recent follow-up. Tumor size and location were analyzed using axial postcontrast T1-weighted images. The location of the tumor relative to the IAC was recorded: entirely in the IAC, entirely within the cerebellopontine angle (CPA), or spanning both. Cochlear intensity was analyzed using axial T2-weighted images. Three circular regions of interest (ROIs) were placed on the T2 images. One ROI was placed within the cochlea of the ear ipsilateral to the tumor, the second ROI within the cochlea of the contralateral ear, and the third ROI within the pons on the same image. The reasoning for the pons as the comparison point is that it has been shown to be consistently hypointense to the cochlea and can be easily visualized on an axial slice in view of both cochleae. An example image of this ROI placement is shown in - Fig. 1.

To standardize MRI signal intensity values among patients, we used an index value by calculating a relative cochlear-to-pontine relative signal intensity (RSI)-a ratio of the signal intensity of the cochlea on one side to the intensity of the pons (method previously described by Kim et al). ${ }^{5}$

Audiograms for baseline and most recent follow-up were reviewed. For both the ipsilateral and contralateral ear, average hearing levels via air conduction measured at 500, 1,000, 2,000, and 4,000 $\mathrm{Hz}$ were collected; the mean of these values was calculated to give a pure tone average (PTA). The four-tone PTA was chosen to capture the high-frequency hearing loss often seen in small and medium sized VSs. Additionally, values for the speech recognition threshold, as well as word recognition score (WRS) and hearing level were collected.

Baseline characteristics were analyzed using descriptive statistics. Chi-square and t-tests were used for categorical and continuous variables, respectively. Paired $t$-tests were used to evaluate the difference between initial and follow-up RSI and PTA. Pearson's correlation coefficient was

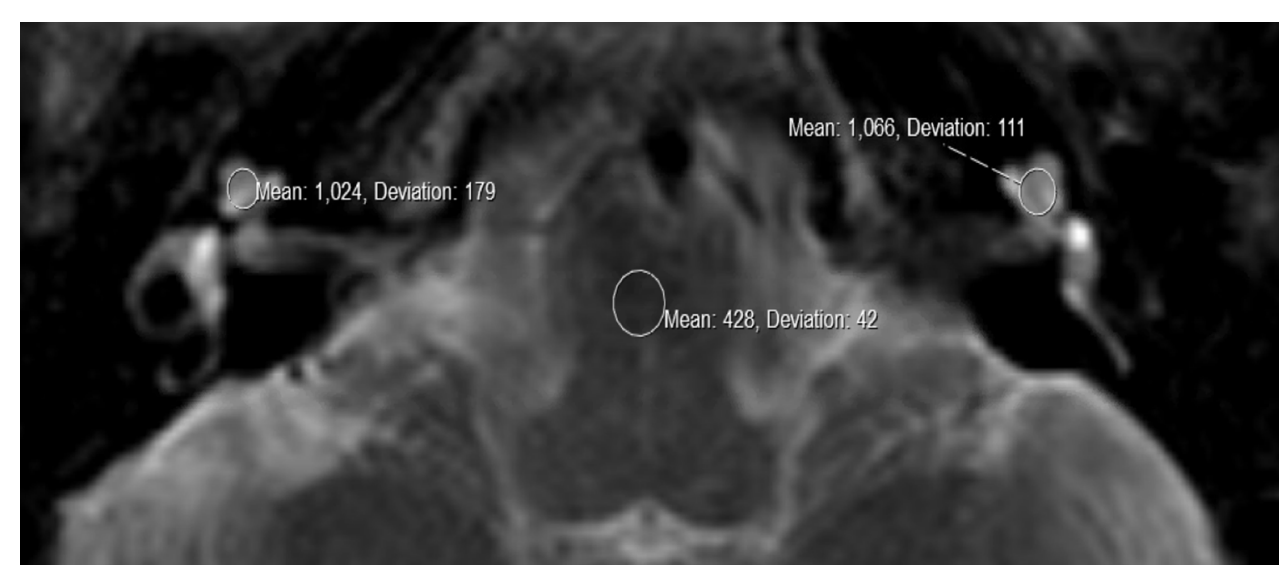

Fig. 1 An axial section of a T2-weighted magnetic resonance imaging (MRI) showing bilateral cochlea and the pons. There is an intracanalicular vestibular schwannoma present in the left ear. To measure cochlear intensity, the SECTRA picture archiving and communication system (PACS) software was used to manually place regions of interest (ROIs) within each cochlea using the ROI circle tool. One ROI was placed within the cochlea of the ear ipsilateral to the tumor (right side of image), the second ROI within the cochlea of the contralateral ear. A third ROI was placed within the pons to obtain a third measurement needed to calculate the relative signal intensity (RSI), the index value we used to compare patients to one another. 
calculated between PTA difference in time and initial RSI. Alpha $<0.05$ was considered statistically significant. The analysis was made in R statistical software 3.6.0.

\section{Results}

An initial search of institutional records found 893 patients with a diagnosis of VS. After excluding cases that did not meet all criteria, 50 patients were included in the final analysis for this study. Demographic and tumor characteristics were overall balanced and are summarized in - Table 1 .

Patients were majority female (54\%) with a mean age of 65.91 years. Majority of tumors were left-sided (72\%), mean tumor size of $119.23 \mathrm{~mm}^{2}$, and most tumors having portions in the CPA and IAC (50\%). No significant differences were found in patient or tumor characteristics between leftand right-sided tumors.

Patient audiometric data was plotted using American Academy of Otolaryngology-Head and Neck Surgery scattergrams. ${ }^{6}$ Plots were made using PTA against WRS, for both unaffected and affected ears ( - Fig. 2). In general, unaffected ears showed lower PTA and higher WRS (i.e., better hearing) scores than those affected ears.

There was a trend in the ipsilateral ear for RSI to increase over time and RSI to decrease in the contralateral ear, though these did not reach statistical significance (ipsilateral $p=0.71$; contralateral $p=0.71$ ) (-Fig. 3A, B). For PTA, the general trend shows both ears having increasing PTA values over time reflecting worsening hearing though this did not reach statistical significance (ipsilateral $p=0.22$; contralateral $p=0.15)$ ( Fig. 3C, D). Both baseline and follow-up PTA values are higher for the ipsilateral ear than the contralateral ear (-Table 2).

When looking at the ipsilateral ear, there is a significant positive correlation between the initial RSI compared with the difference in the PTA between baseline and follow-up $(R=0.28, p=0.048)$ ( - Fig. 4). There was a negative trend for the contralateral ear which showed no significance.

There was also no significant correlation for either ipsilateral or contralateral ears when comparing change in PTA and change in RSI over time from the baseline to follow-up time points (ipsilateral $R=-0.17, p=0.32$; contralateral $R=-0.18$, $p=0.3)($-Fig. 5A, B).

\section{Discussion}

This study examined the relationship between hearing loss and MRI findings, specifically how the cochlea appeared on T2 imaging in patients with VSs. The ultimate goal was to see if there was a relationship between PTA and RSI over time. As expected, there was a trend for the PTA to increase from baseline to follow-up, representing worsening hearing,

Table 1 Patient demographics and tumor characteristics

\begin{tabular}{|c|c|c|c|c|}
\hline & Level & Left ear & Right ear & $p$ \\
\hline$N$ & & 36 & 14 & \\
\hline \multirow[t]{2}{*}{ Age (mean [SD]) } & & $65.75 y$ & $66.07 \mathrm{y}$ & \multirow[t]{2}{*}{0.926} \\
\hline & & $(11.18)$ & $(10.12)$ & \\
\hline \multirow[t]{2}{*}{ Sex $(\%)$} & $\mathrm{F}$ & $18(50.0)$ & $9(64.3)$ & \multirow[t]{2}{*}{0.552} \\
\hline & $\mathrm{M}$ & $18(50.0)$ & $5(35.7)$ & \\
\hline \multirow[t]{2}{*}{ Size (mean [SD]) } & & $97.78 \mathrm{~mm}^{3}$ & $140.68 \mathrm{~mm}^{3}$ & \multirow[t]{2}{*}{0.186} \\
\hline & & $(93.00)$ & $(121.27)$ & \\
\hline \multirow[t]{3}{*}{ Location (\%) } & Both & $16(44.4)$ & $9(64.3)$ & \multirow[t]{3}{*}{0.408} \\
\hline & CPA & $1(2.8)$ & $0(0.0)$ & \\
\hline & IAC & $19(52.8)$ & $5(35.7)$ & \\
\hline T2 ipsilateral (mean [SD]) & & $\begin{array}{l}704.61 \\
(671.38)\end{array}$ & $653.50(210.04)$ & 0.782 \\
\hline T2 contralateral (mean [SD]) & & $\begin{array}{l}709.69 \\
(548.55)\end{array}$ & $512.00(157.01)$ & 0.193 \\
\hline T2 pons (mean $[S D]$ ) & & $\begin{array}{l}379.17 \\
(333.77)\end{array}$ & $357.50(177.15)$ & 0.819 \\
\hline RSI ipsilateral initial (mean [SD]) & & $1.87(0.47)$ & $1.92(0.39)$ & 0.729 \\
\hline RSI contralateral initial (mean [SD]) & & $1.89(0.40)$ & $1.61(0.58)$ & 0.054 \\
\hline Ipsilateral PTA initial (mean [SD]) & & $\begin{array}{l}49.83 \mathrm{~dB} \\
(19.65)\end{array}$ & $49.55 \mathrm{~dB}(21.81)$ & 0.966 \\
\hline Contralateral PTA initial (mean [SD]) & & $18.30 \mathrm{~dB}(9.27)$ & $21.07 \mathrm{~dB}(10.67)$ & 0.367 \\
\hline
\end{tabular}

Abbreviations: CPA, cerebellopontine angle; dB, decibels; IAC, internal auditory canal; PTA, pure tone average; RSI, relative signal intensity; SD, standard deviation. 


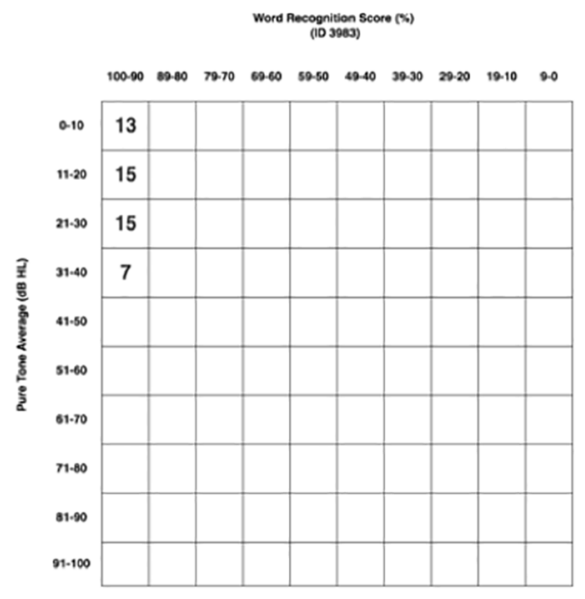

Unaffected ear

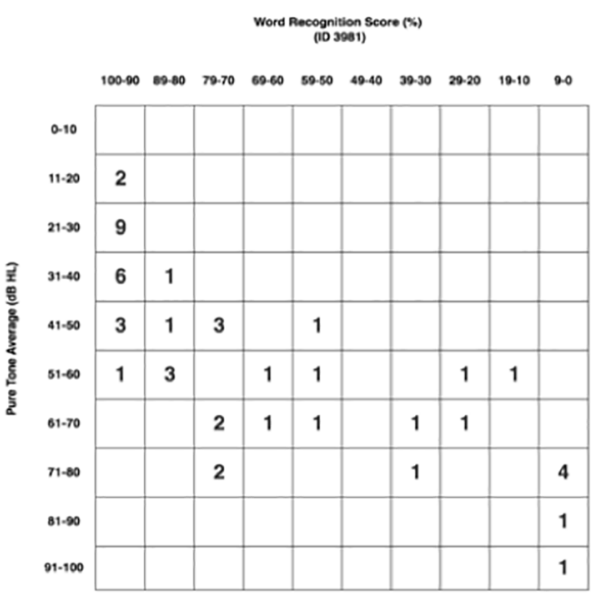

Affected ear

Fig. 2 American Academy of Otolaryngology-Head and Neck Surgery scattergrams for both unaffected and affected ears are shown. Word recognition score (WRS) (\%) is shown on the $x$-axis and pure tone average (PTA (dB) on the $y$-axis. The frequency of patients whose audiometric data places them within those PTA and WRS parameters is represented numerically within the squares.
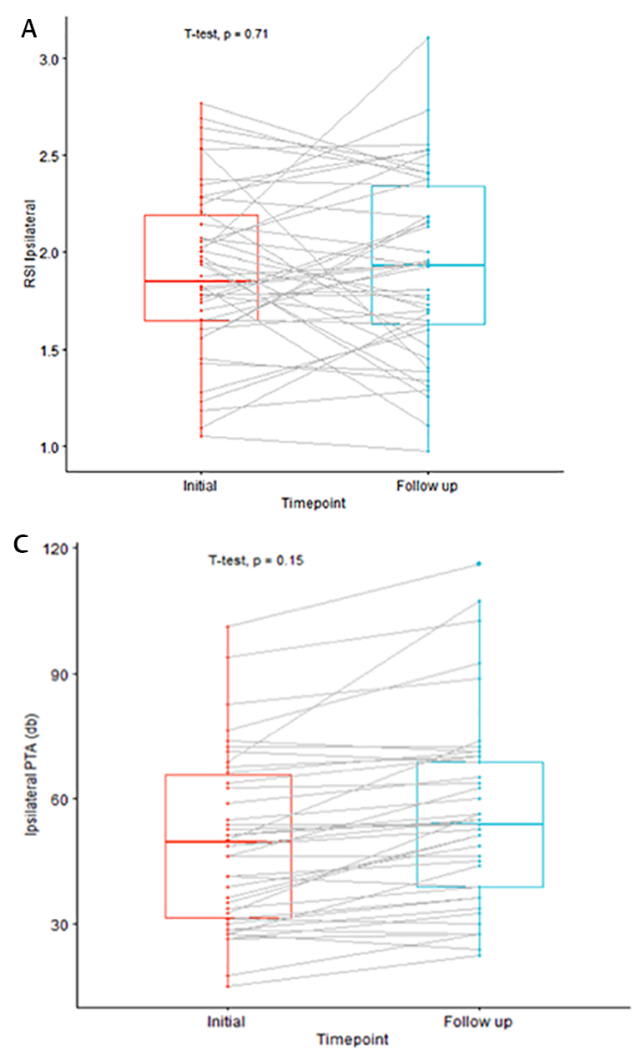
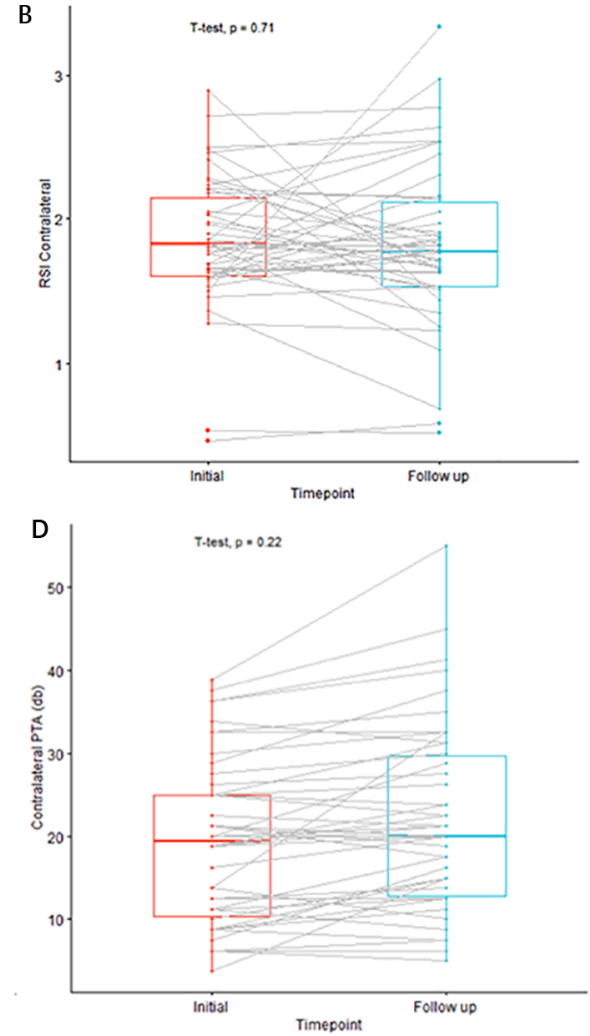

Fig. 3 Box and whisker plots of (A) relative signal intensity (RSI) ipsilateral, (B) RSI contralateral, at initial visit and at their first follow-up visit, (C) pure tone average (PTA) ipsilateral, and (D) PTA contralateral, at initial visit and at their first follow-up visit. The gray lines between the initial and the follow-up plots, identify the change in each individual patient. $t$-Tests compared the means of each group. $p$-Values are shown in each plot.

for both the ipsilateral and contralateral ear with a greater degree of change in the ipsilateral ear. For RSI, the direction of the trend depended on which ear was being studied. RSI was shown to increase over time for affected ears, and decreased for unaffected ears ( - Fig. $\mathbf{3}$ ).

When correlating the RSI at the initial time point with the change in PTA over time, there was a significant correlation seen in the ipsilateral ear ( - Fig. 4), without an associated correlation in the contralateral ear. This finding suggests that patients with changes to the cochlear T2 signal in their affected ear on initial imaging may have larger changes to their hearing over time. Previous literature has found that in patients affected with VSs, three-dimensional fluid-attenuated inversion recovery (FLAIR) cochlear intensity increases with 

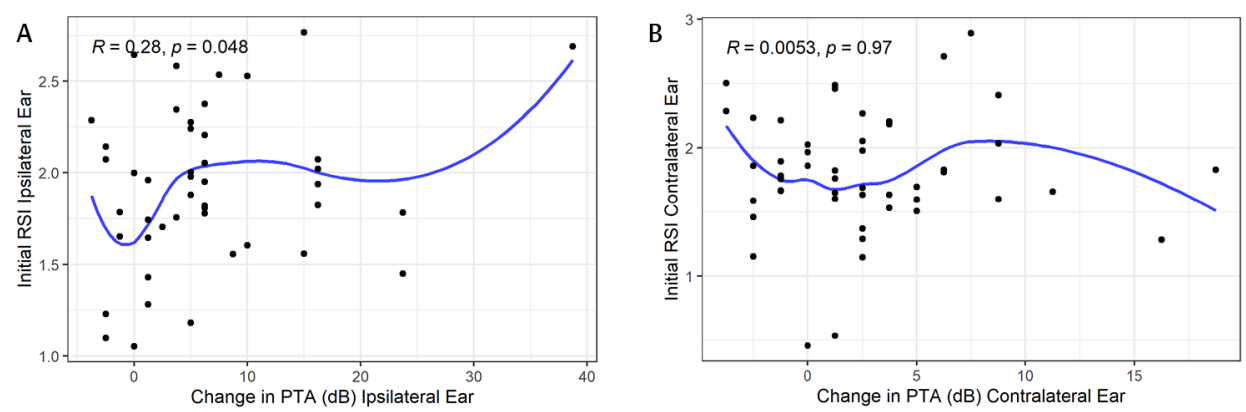

Fig. 4 Scatterplots showing the relationship between the percentage of the initial relative signal intensity (RSI) for an ear compared with the change in pure tone average (PTA). (A) Ipsilateral ear and (B) the contralateral ear. Pearson's $R$ values and $p$-values are labeled in each graph.

Table 2 Mean differences for audiometry and MRI signal from baseline to follow-up

\begin{tabular}{|l|c|c|}
\hline & Mean & SD \\
\hline \multicolumn{2}{|l|}{ Difference in audiometry baseline to follow-up (dB) } \\
\hline Ipsilateral PTA & $6.075 \mathrm{~dB}$ & $8.089148 \mathrm{~dB}$ \\
\hline Contralateral PTA & $2.575 \mathrm{~dB}$ & $4.686675 \mathrm{~dB}$ \\
\hline \multicolumn{2}{|c|}{ Difference in MRI baseline to follow-up (ratio) } \\
\hline Ipsilateral RSI & 0.02778505 & 0.4133739 \\
\hline Contralateral RSI & -0.00914956 & 0.5312379 \\
\hline
\end{tabular}

Abbreviations: $\mathrm{dB}$, decibels; MRI, magnetic resonance imaging; PTA, pure tone average; $\mathrm{RSI}$, relative signal intensity; SD, standard deviation.

greater hearing loss. ${ }^{5}$ Thus, the RSI of affected ears increasing between follow-ups in our cohort was a consistent finding. However, to our knowledge no studies have attempted to compare the MRI signal and audiometry between multiple time points.

When analyzing association between changes in RSI with changes in PTA from our first data set to the follow-up data set, we were unable to find a significant relationship for either ear (-Fig. 5). This temporal relationship was the one we were most interested in in terms of being able to form a predictive relationship between MRI signal and hearing loss. Lack of correlation found here could indicate a need for further data points and follow-up to create a more detailed relationship.

VSs are well known to be slow growing which makes observation a reasonable option for patient management. There is risk that hearing will worsen and tumor size will increase during the observation period, which may make these patients not ideal candidates for hearing preservation surgery if they decide to switch management. ${ }^{7-9}$ For this reason, trying to associate imaging findings in patients with VS with hearing loss has been the subject of many studies in recent years. Tumor characteristics such as tumor size, growth rate, and laterality have not shown to be consistent predictors of clinical symptoms in affected patients. ${ }^{4,10,11}$

MRI is sensitive to the perilymphatic protein content within the cochlea. It has been well reported in the literature that patients with VSs have increased levels of protein in the perilymph of their affected ears as compared with ears that are unaffected. ${ }^{12-14}$ Intralabyrinthine signal changes on MR and any attached prognostic value has been of interest to surgeons since the technology was available to detect such variations in intensity within the cochlea. ${ }^{15}$ The exact mechanism is not quite clear, however, one proposed explanation is that tumors cause compression of the cochlear nerve, resulting in interference with the neuroaxonal transport of proteins. ${ }^{14} \mathrm{Kim}$ et al found that there was a significant correlation between the T2 FLAIR signal and the degree of hearing impairment, ${ }^{5}$ while others suggest the relationship between FLAIR and level of hearing impairment is weaker. ${ }^{5,16}$

MRI has also been used to look at endolymphatic hydrops (EH), another phenomenon found in the affected cochlea of VS patients. ${ }^{17,18}$ Eliezer et al ${ }^{19}$ describes a correlation between EH and hearing loss. They found the utricle volume imaged using T2-weighted fast imaging employing steady-state acquisition with cycle phase was correlated with the patient's hearing loss in a VS patient series. Karch-Georges et $\mathrm{al}^{20}$ researched T2 MRI at 3 Tesla and found a third of their cohort had their affected cochlea associated with saccular dilation, another radiographic sign of $\mathrm{EH}$. These last two aforementioned studies also suggest treatment that can decrease EH could hypothetically be used to result in better hearing. ${ }^{19,20}$

The retrospective nature of this study meant that we were unable to standardize the time interval in between follow-ups, as well as the interval between MRI and audiometry. Our criteria for included patients focused on having two sets of temporally linked audiometric and radiographic data-one for baseline and a follow-up. To mitigate the effect of these variable intervals, future studies would need to be prospective in nature with these intervals standardized.

Another disadvantage of the retrospective design was that it resulted in a final patient subset (50) being much smaller portion than the sample of patients initially found to have a diagnosis of VS, with the primary reason for exclusion being lack of adequate radiographic and audiometric data to have two complete temporally linked sets of data.

For measurement of the RSI, the ROIs had to be manually placed onto the images which introduced an element of human error. Future studies would ideally have at least one neuroradiologist participate in the placement of the ROIs and interpretation of the images; more than one would increase the interrater reliability of such results.

Our goal with this study was to find a relationship between hearing loss and MRI signals, and see if that could be extrapolated to create a predictive model. Two time points of data cannot be used reliably to create a true predictive model and 

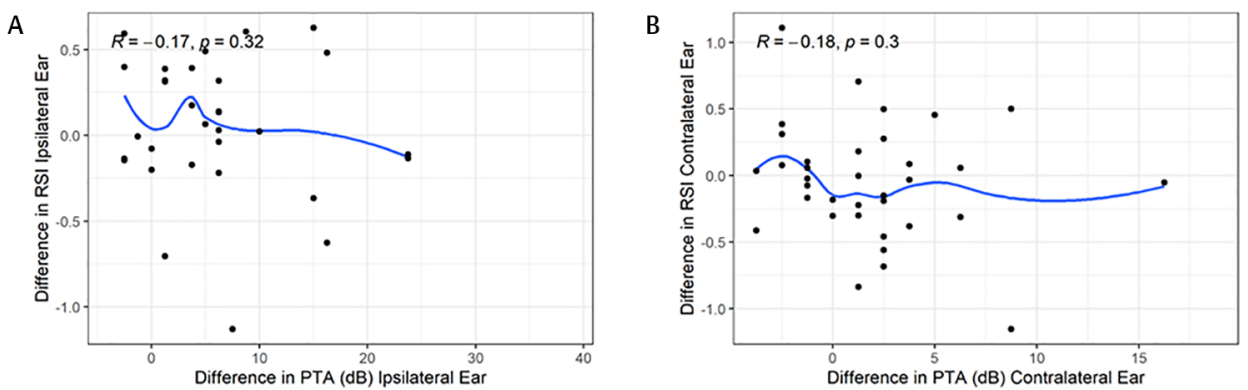

Fig. 5 Scatterplots showing changes in pure tone average (PTA) with changes in relative signal intensity (RSI), from first data set to follow-up. (A) The ipsilateral ear and (B) the contralateral ear. Pearson's $R$ values and $p$-values are labeled in each graph.

has limited prognostic value within itself. However, as the basis of a pilot study, we were able to show that it is possible to use these measurements of audiometric and MRI to form a correlation over time. If these results were to be expanded with a larger prospective study, a trend with more clinical significance could be explored. This could allow providers to have a better idea of when the optimal time to intervene is as they could do serial MRI, trend those, and correlate that with the corresponding hearing loss trend to gauge when the optimal timeframe for surgical or radiosurgical intervention would be to best preserve patient hearing.

Another aspect that could be interesting to investigate would be to compare the signal from both T2 and FLAIR images for the same patients. For this pilot retrospective study, FLAIR signals could not be used due to the MRI IAC protocol lacking thin cut FLAIR images through the cochlea. Additionally, images were taken on a variety of scanners and differences in scanner software and image analysis may also impact these results. Future studies would include more rigorous control and standardization of the imaging technique to ensure that variables such as machine manufacturer and sequence were constant for all patients.

\section{Conclusion}

There appears to be a positive trend between initial $\mathrm{T} 2$ cochlear signal and hearing changes in the affected ears of patients with VS. Moving forward, prospective studies with larger cohorts, consistent serial data sets, and more standardization in MR usage with the inclusion of a neuroradiologist on the team are ideally needed to elucidate the relationship between our variables and determine their clinical significance for a larger and more generalizable patient population.

\section{Conflict of Interest}

None declared.

\section{References}

1 Matthies C, Samii M. Management of 1000 vestibular schwannomas (acoustic neuromas): clinical presentation. Neurosurgery 1997;40(1):1-9, discussion 9-10

2 Kondziolka D, Mousavi SH, Kano H, Flickinger JC, Lunsford LD. The newly diagnosed vestibular schwannoma: radiosurgery, resection, or observation? Neurosurg Focus 2012;33(3):E8
3 Lin EP, Crane BT. The management and imaging of vestibular schwannomas. AJNR Am J Neuroradiol 2017;38(11):2034-2043

4 Yoshimoto Y. Systematic review of the natural history of vestibular schwannoma. J Neurosurg 2005;103(1):59-63

$5 \mathrm{Kim}$ DY, Lee JH, Goh MJ, et al. Clinical significance of an increased cochlear 3D fluid-attenuated inversion recovery signal intensity on an MR imaging examination in patients with acoustic neuroma. AJNR Am J Neuroradiol 2014;35(9):1825-1829

6 Oghalai JS, Jackler RK. New web-based tool for generating scattergrams to report hearing results. Otolaryngol Head Neck Surg 2016;154(6):981

7 Stangerup SE, Thomsen J, Tos M, Cayé-Thomasen P. Long-term hearing preservation in vestibular schwannoma. Otol Neurotol 2010;31(2):271-275

8 Chiluwal AK, Rothman A, Svrakic M, Dehdashti AR. Surgical outcome in smaller symptomatic vestibular schwannomas. Is there a role for surgery? Acta Neurochir (Wien 2018;160(11):2263-2275

9 Akpinar B, Mousavi SH, McDowell MM, et al. Early radiosurgery improves hearing preservation in vestibular schwannoma patients with normal hearing at the time of diagnosis. Int J Radiat Oncol Biol Phys 2016;95(2):729-734

10 Nadol JB Jr, Diamond PF, Thornton AR. Correlation of hearing loss and radiologic dimensions of vestibular schwannomas (acoustic Neuromas. Am J Otol 1996;17(2):312-316

11 Tierney PA, Chitnavis BP, Sherriff M, Strong AJ, Gleeson MJ. The relationship between pure tone thresholds and the radiological dimensions of acoustic neuromas. Skull Base Surg 1998;8(3):149-151

12 Silverstein $\mathrm{H}$, Schuknecht HF. Biochemical studies of inner ear fluid in man. Changes in otosclerosis, Meniere's disease, and acoustic neuroma. Arch Otolaryngol 1966;84(4):395-402

13 O'Connor AF, France MW, Morrison AW. Perilymph total protein levels associated with cerebellopontine angle lesions. Am J Otol 1981;2(3):193-195

14 Bhadelia RA, Tedesco KL, Hwang S, et al. Increased cochlear fluid-attenuated inversion recovery signal in patients with vestibular schwannoma. AJNR Am J Neuroradiol 2008;29(4):72072310.3174/ajnr.A0968

15 Somers T, Casselman J, de Ceulaer G, Govaerts P, Offeciers E. Prognostic value of magnetic resonance imaging findings in hearing preservation surgery for vestibular schwannoma. Otol Neurotol 2001;22(1):87-94

16 Yamazaki M, Naganawa S, Kawai H, Nihashi T, Fukatsu H, Nakashima T. Increased signal intensity of the cochlea on preand post-contrast enhanced 3D-FLAIR in patients with vestibular schwannoma. Neuroradiology 2009;51(12):855-863

17 Lassaletta L, Calvino M, Morales-Puebla JM, et al. Biomarkers in vestibular schwannoma-associated hearing loss. Front Neurol 2019;10:978

18 Roosli C, Linthicum FH Jr, Cureoglu S, Merchant SN. Dysfunction of the cochlea contributing to hearing loss 
in acoustic neuromas: an underappreciated entity. Otol Neurotol 2012;33(3):473-480

19 Eliezer M, Poillon G, Maquet C, et al. Sensorineural hearing loss in patients with vestibular schwannoma correlates with the presence of utricular hydrops as diagnosed on heavily T2-weighted MRI. Diagn Interv Imaging 2019;100(5):259-268
20 Karch-Georges A, Veillon F, Vuong H, et al. MRI of endolymphatic hydrops in patients with vestibular schwannomas: a case-controlled study using non-enhanced T2-weighted images at 3 Teslas. Eur Arch Otorhinolaryngol 2019;276(6):1591-1599 